\title{
Perspectives for the study of quarkonium production in pp collisions with the ALICE muon spectrometer
}

\author{
Livio BIANCHI for the ALICE Collaboration \\ Universita' \& INFN di Torino \\ E-mail: lbianchi@to.infn.it
}

\begin{abstract}
One of the main physics goals of the muon spectrometer of the ALICE experiment is the study of quarkonium resonances at forward rapidity $(2.5<y<4)$ in a wide transverse momentum range (from 0 to tens of $\mathrm{GeV}$ ). The quarkonium production mechanism in proton-proton collisions is at present an open issue: several theoretical predictions are available, but they need to be confirmed by experimental data. These kind of studies are very interesting also to provide a proper normalization for the study of hot nuclear effects in $\mathrm{Pb}-\mathrm{Pb}$ collisions. Data from pp collisions at $\sqrt{ } s=7 \mathrm{TeV}$ will be soon available from the LHC. The muon spectrometer of ALICE will be able to measure quarkonia cross sections, transverse momentum distribution and polarization after a few months of data taking.
\end{abstract}

XLVIII International Winter Meeting on Nuclear Physics in Memoriam of Ileana Iori 25-29 January 2010

Bormio, Italy

\footnotetext{
* Speaker.
} 


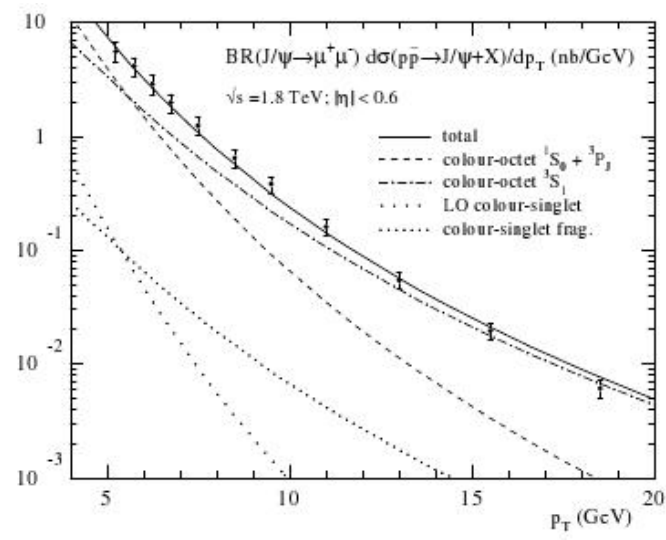

(a) $\mathrm{CDF}: J / \psi \rightarrow \mu^{+} \mu^{-}[1]$

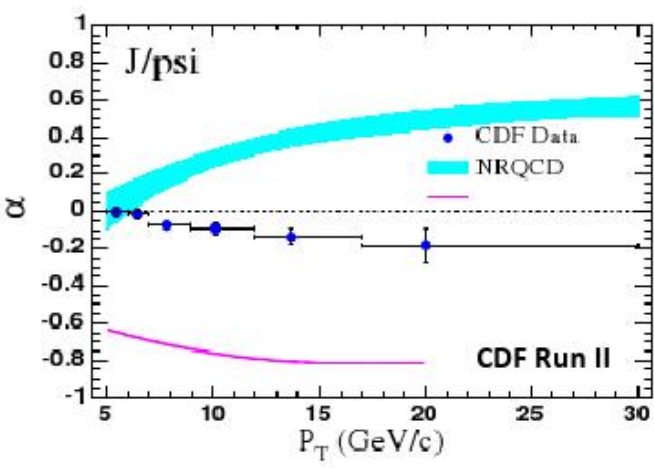

(b) CDF: polarization [2]

Figure 1: $J / \psi$ differential cross-section and polarization as measured by the CDF collaboration

\section{The Quarkonium production puzzle}

The production of any given quarkonium state [1] is believed to be factorisable into two parts: the first, where a heavy quark and antiquark pair is produced, is driven by perturbative QCD; the second, concerning the formation of a physical bound state, is under the auspices of nonperturbative QCD and models have been proposed to explain the properties of quarkonium production.

With precise measurements of quarkonia and a large quantity of data available, the mechanism underlying the production of these resonances still remains elusive, being more complicated than initially expected.

The Colour Singlet Model (CSM) of quarkonium production enjoyed some success before CDF measured an excess of direct $J / \psi$ production more than an order of magnitude greater than predicted; moreover the $p_{T}$ dependence provided by the model was uncorrect (see Figure 1a). The Colour Octet Mechanism (COM) was proposed as a solution to this problem, suggesting that the heavy quark pairs could evolve into quarkonium states with particular quantum numbers through radiation of soft gluons during hadronisation. CSM and COM are two different truncations of the effective field theory called NRQCD, which provides the formalism for the factorization approach. Other phenomenological models are the CEM (Color Evaporation Model - see Section 3) and the so-called CSM+s-channel cut, which is a promizing approach for the understanding of preliminary data from Phenix.

Despite the successes of COM to describe the observed $p_{T}$ differential cross-section, recent measurements at $\mathrm{CDF}$ and $\mathrm{D} \emptyset$ show disagreement with COM predictions on polarization (see Figure $1 \mathrm{~b}$ and Section 4).

\section{The ALICE Forward Muon Spectrometer}

ALICE [3] is the dedicated heavy-ion experiment at the LHC. It can be seen as the integration of a central barrel part (which covers mid-rapidity, $|y|<0.9$ ) with an arm dedicated to the detection 
of muons, the Forward Muon Spectrometer.

The angular acceptance of the Forward Spectrometer $[4,5]$ covers the range $2^{\circ}-9^{\circ}$ corresponding to a pseudorapidity range from 2.5 to 4 . Since it covers the forward direction, its design is similar to solutions used in fixed target experiments [6]. The important difference is that the forward hadron flow cannot be absorbed in a plug because the beam pipe passes through the experiment. Instead, the particles have to be absorbed in a dense beam shield $(\mathrm{Pb}$ and $\mathrm{W})$ decreasing the hit rates on the detector planes to acceptable levels.

The front absorber, being placed between the interaction point and the first tracking chamber, has a total of $10 \lambda_{I}$ and consists of carbon, concrete and steel. The outer surface of the absorber is covered by heavy materials ( $\mathrm{W}$ and $\mathrm{Pb}$ ), in order to shield the central barrel detectors (mainly the TPC) against background particles. The tracking system consists of five stations with two planes each, made of very thin $\left(\sim 5 \% X_{0}\right)$ cathode pad tracking stations. To limit the occupation rate to a maximum of $5 \%$ in central $\mathrm{Pb}-\mathrm{Pb}$ collisions, the full set of chambers has more than 1 million channels. One tracking station is placed inside a dipole magnet with a maximum field of $0.7 \mathrm{~T}$. The momentum of the muons is determined from a combination of deflection angle and sagitta measurement. The muon filter, a $7 \lambda_{I} \mathrm{Fe}$ absorber wall, suppresses the remaining hadron rate on the 4 planes of single-gap RPCs of the trigger system, which have a high time resolution and provide a level-0 muon and dimuon triggers to the ALICE central trigger system in $\sim 800 \mathrm{~ns}$.

\section{Quarkonia production in pp collisions}

In the forward muon arm, $J / \psi$ production at $\sqrt{s}=14 \mathrm{TeV}$ has been simulated using the Color Evaporation Model (CEM). In this model the quarkonium production cross section is a measurable fraction $\left(F_{C}\right)$ of all $Q \bar{Q}$ pairs below the $H \bar{H}$ threshold (where $H$ is the lowest mass heavy flavour hadron), without any constraint on the color or spin of the final state. The $Q \bar{Q}$ pair then neutralizes its color by interaction with the collision-induced color field.

At leading order, the production cross section of a quarkonium state $\mathrm{C}$ in a $\mathrm{AB}$ collision is:

$$
\sigma_{C}^{C E M}=F_{C} \sum_{i j} \int_{4 m_{Q}^{2}}^{4 m_{H}^{2}} d \hat{s} \int d x_{1} d x_{2} f_{i / A}\left(x_{1}, \mu^{2}\right) f_{j / B}\left(x_{2}, \mu^{2}\right) \hat{\sigma}_{i j}(\hat{s}) \delta\left(\hat{s}-x_{1} x_{2} s\right)
$$

where $\mathrm{A}$ and $\mathrm{B}$ can be any hadron or nucleus, $i j=q \bar{q}$ or $g g, \hat{\sigma}_{i j}(\hat{s})$ is the $i j \rightarrow Q \bar{Q}$ subprocess cross section and $f_{i / A}\left(x_{1}, \mu^{2}\right)$ is the parton density in the hadron or nucleus. Finally, $\mathrm{s}$ and $\hat{s}$ are respectively the hadronic and partonic center of mass energies.

The results presented here have been obtained with the MRST HO PDF, with $m_{c}=1.2 \mathrm{GeV} / \mathrm{c}^{2}$ and $\mu=2 m_{c}$ [7]. The calculation gives a $J / \psi$ total cross-section of $53.9 \mu \mathrm{b}$, including the feed-down from higher mass resonances.

A typical data taking period of one year (assuming $10^{7} \mathrm{~s}$ running time) at $L=3 \cdot 10^{30} \mathrm{~cm}^{-2} \mathrm{~s}^{-1}$ corresponds to an integrated luminosity of $30 \mathrm{pb}^{-1}$. With these inputs, the expected $J / \psi$ statistics is $\sim 2.8 \cdot 10^{6}$ events [8]. The background under the $J / \psi$ peak (see Figure 2) is dominated by correlated decays of heavy flavours, but is anyway expected to be quite small $(\mathrm{S} / \mathrm{B}=12)$. It will be possible to study the transverse momentum distribution of the $J / \psi$ with negligible statistical errors up to at least $p_{T}=20 \mathrm{GeV} / \mathrm{c}$.

For what concerns the $\Upsilon(1 S)$ an amount of $\sim 2.7 \cdot 10^{4}$ events is expected in one year of data taking; 


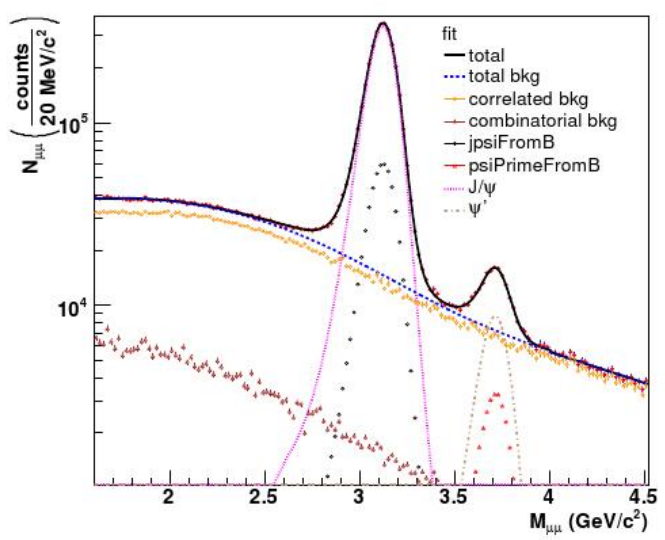

(a) Charmonium mass spectrum

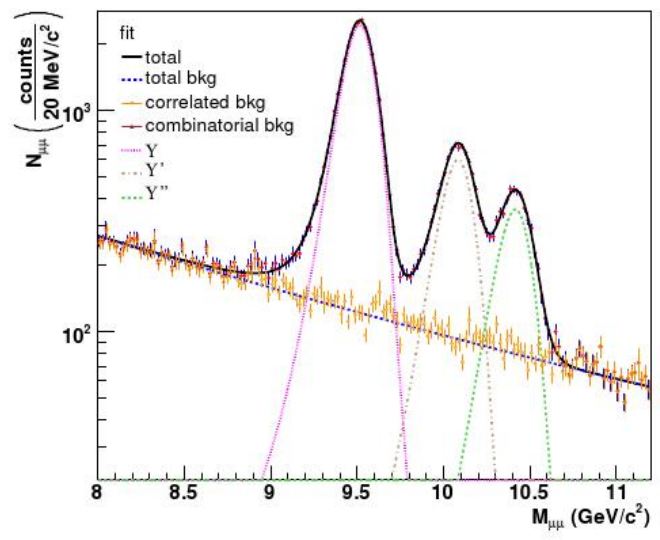

(b) Bottomonium mass spectrum

Figure 2: Opposite sign dimuon mass spectra expected for $\sqrt{s}=14 \mathrm{TeV}$ proton-proton collisions at nominal luminosity $\left(3 \cdot 10^{30} \mathrm{~cm}^{-2} \mathrm{~s}^{-1}\right)$ in $10^{7} \mathrm{~s}[8]$

this statistics will allow, in addition to the integrated cross section measurement, a study of the $p_{T}$ distribution.

\section{Polarization}

The polarization of a quarkonium state can be determined by measuring angular asymmetries of the decaying products. The full angular dependence of the decay width is:

$$
\frac{1}{\Gamma} \frac{d \Gamma}{d \cos \theta d \phi}=1+\alpha \cos ^{2} \theta+\mu \sin (2 \theta) \cos \phi+\frac{v}{2} \sin ^{2} \theta \cos (2 \phi)
$$

while usually (but not always properly), the $\theta$ dependence is the only considered:

$$
\frac{d \Gamma}{d \cos \theta} \propto \frac{3}{2(\alpha+3)}\left(1+\alpha \cos ^{2} \theta\right)
$$

The $\alpha$ parameter is related to the fraction of $Q \bar{Q}$ observed in the longitudinal and transverse spin states:

$$
\alpha=\left(\sigma_{T}-2 \sigma_{L}\right) /\left(\sigma_{T}+2 \sigma_{L}\right)
$$

and the measured value depends on the polarization axis chosen (e.g. Helicity or Collins-Soper [9]).

The evolution with respect to $p_{T}$ of the $\alpha$ parameter is a possible way to discriminate among the different production models, since it is strongly dependent on the model itself.

With the expected statistics at $\sqrt{s}=14 \mathrm{TeV}$ and nominal luminosity (see Section 3), it will be possible to carry out a detailed analysis of the $p_{T}$ dependence of the $\alpha$ parameter for both inclusive $J / \psi$ and $\Upsilon$. Figure 3 shows the $\alpha$ parameter reconstruction capability in an integrated-over- $p_{T}$ approach (a) and in four $p_{T}$ bins (b) for the $\Upsilon(1 S)$. With this statistics an analysis of the full angular distribution will also be achievable. 


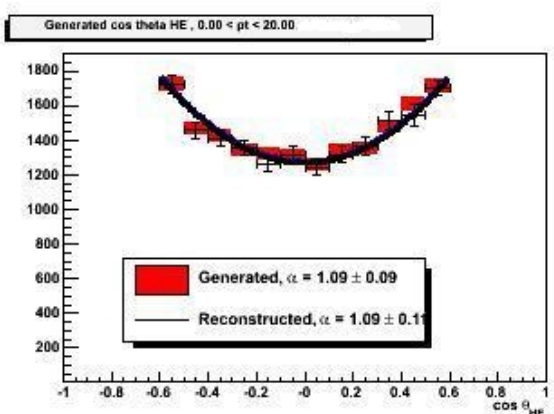

(a) Polarization parameter reconstruction from a fit to the $\cos \theta$ distribution. Helicity reference frame, $p_{T}$ range: $0-20 \mathrm{GeV} / \mathrm{c}$

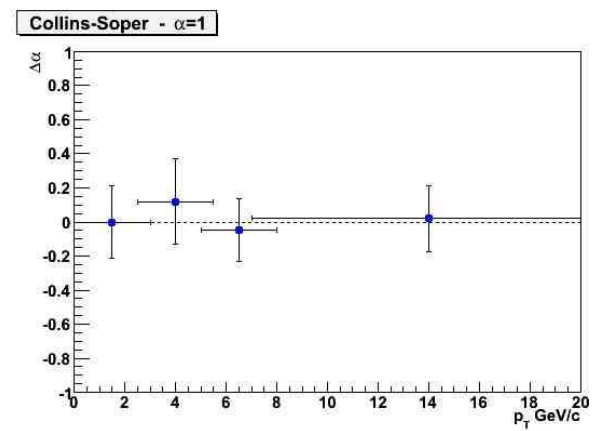

(b) Difference between $\alpha_{\text {rec }}$ and $\alpha_{g e n}$ in four $p_{T}$ bins in the Collins-Soper reference frame

Figure 3: $\Upsilon(1 S)$ polarization study in the ALICE Forward Muon Spectrometer

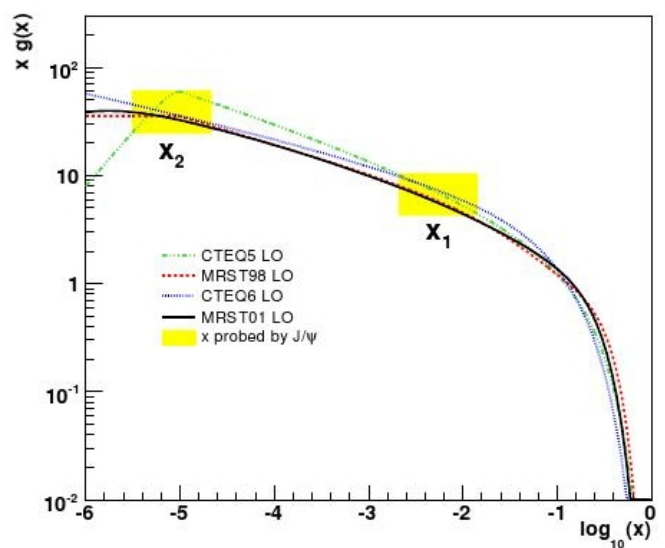

(a) $x$ regions probed by the $J / \psi$

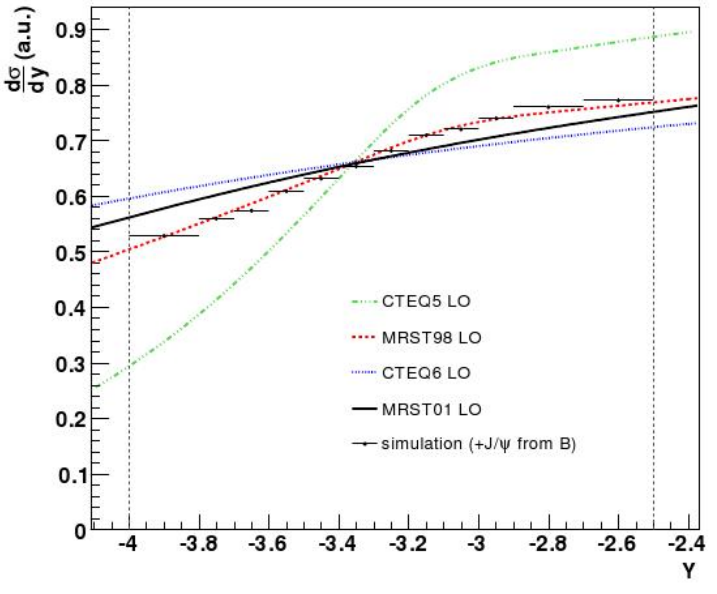

(b) $J / \psi$ rapidity distributions obtained with four different PDF sets and simulation results

Figure 4: Low- $x$ gluon PDF study from $J / \psi$ produced at $\sqrt{s}=14 \mathrm{TeV}$ in 1 year of data taking at nominal luminosity [8]

\section{Low- $x$ gluon Parton Distribution Function}

The PDF for gluons is poorly known for $x$ around and below $10^{-5}$, where the various calculations show significant differences, having to rely on extrapolations. Leading order calculations show that in pp collisions at $\sqrt{s}=14 \mathrm{TeV}, J / \psi$ with $y>3$ are produced by gluons carrying a fraction $x<10^{-5}$ of the proton momentum. CEM calculations at $\sqrt{s}=14 \mathrm{TeV}$ and nominal luminosity [8] show that, analyzing the y distribution for $J / \psi$ and $\Upsilon$ collected in 1 year, we can discriminate among different gluon distribution functions in the region $x<10^{-5}$, which accessible for the muon spectrometer (see Figure 4a). 


\section{Perspectives for the first high-energy run}

The ALICE experiment has started its data taking during December 2009 at $900 \mathrm{GeV}$ and the Forward Muon Spectrometer has collected some thousands of tracks. Starting from March 2010, the LHC should deliver several months of pp collisions at $\sqrt{s}=7 \mathrm{TeV}$. Estimating to have $L \sim 2 \cdot 10^{29} \mathrm{~cm}^{-2} \mathrm{~s}^{-1}$ in ALICE and a running efficiency of $\sim 20 \%$ as a conservative value, it might be possible (with single-muon trigger) to collect $2-3 \cdot 10^{4} \mathrm{~J} / \psi$ in the spectrometer. In this scenario a measurement of the $p_{T}$ distribution and an estimate of the polarization value integrated over $p_{T}$ could be within reach. For what concerns $\psi(2 S)$ and $\Upsilon(1 S)$ some hundred events could be collected, which are enough for an estimate of the integrated cross section.

\section{Conclusions}

The Forward Muon Spectrometer of the ALICE experiment is ready to take data in pp collisions at high energy. In a pp run at $\sqrt{s}=14 \mathrm{TeV}$ and $L=3 \cdot 10^{30} \mathrm{~cm}^{-2} \mathrm{~s}^{-1}$ (nominal running scenario for ALICE), differential cross sections and polarization for all quarkonia resonances will be measured. It will also be possible to access gluon PDF at low-x.

During the foreseen lower-energy scenario $(\sqrt{s}=7 \mathrm{TeV})$ the luminosity will probably be smaller (e.g. $L \sim 2 \cdot 10^{29} \mathrm{~cm}^{-2} \mathrm{~s}^{-1}$ ). In this case the $p_{T}$ distribution and an integrated value of polarization could be achievable for the $J / \psi$ and integrated cross sections might be accessible for $\Upsilon(1 S)$ and $\psi(2 S)$.

\section{References}

[1] N. Brambilla et al., Heavy Quarkonium Physics, Addison-Wesley, 2005

[2] CDF Collaboration, Polarization of $J / \psi$ and $\psi(2 S)$ mesons produced in p anti-p collisions at $\sqrt{s}=$ 1.96 TeV, Phys.Rev.Lett.99:132001,2007

[3] ALICE collaboration, The ALICE experiment at the CERN LHC, JINST 3 (2009) S08002

[4] ALICE Collaboration, TDR of the MUON Spectrometer, CERN/LHCC 99-2 (1999)

[5] ALICE Collaboration, Alice: Physics performance report, volume II, Journal of Physics G, 32(10):1295-2040 (2006)

[6] NA50 Collaboration, J/Psi and Drell-Yan cross-sections in Pb-Pb interactions at $158 \mathrm{GeV} / \mathrm{c}$ per nucleon, Physics Letters B410 (1997) 327

[7] M. Bedjidian et al., Hard probes in heavy ion collisions at the LHC: heavy flavour physics, CERN Yellow Report CERN-2004-009-C

[8] D. Stocco et al.,Quarkonia detection with the ALICE Muon Spectrometer in pp collisions at $\sqrt{s}=14$ $\mathrm{TeV}$, ALICE-INT-2006-029

[9] J.C. Collins and D.E. Soper,Angular distribution of dileptons in high-energy hadron collisions, Phys. Rev. D16, 2219 (1977)

[10] The ALICE Collaboration, First proton-proton collisions at the LHC as observed with the ALICE detector: measurement of the charged particle pseudorapidity density at $\sqrt{s}=900 \mathrm{GeV}$, Eur. Phys. J. C (2010) 65: 111-125 